\section{A comparative analysis of national food recall systems}

\author{
Gaetano Liuzzo, ${ }^{1}$ Andrea Serraino, ${ }^{2}$ \\ Federica Giacometti, ${ }^{2}$ Elena Bonfante ${ }^{2}$ \\ ${ }^{1}$ Azienda Unità Sanitaria Locale di \\ Modena - Distretto di Carpi; \\ 2Dipartimento di Scienze Mediche \\ Veterinarie, Alma Mater Studiorum - \\ Università di Bologna, Ozzano \\ dell'Emilia, Italy
}

\section{Abstract}

Food recall and withdrawal is a fundamental tool for risk management and different countries stated the mandatory application of a system for food; the present work is an analysis of different systems applied in different countries. The main objective of analysed systems is the consumer's health protection through an audit system and the application of system to rapidly recall/withdraw food on the part of producers. The comparative analysis of different national systems [i.e. European Union (EU), Australia, Canada, US and China] shows differences both of the terminological and legal aspects; the words recall and withdrawal have different meaning in EU legislation than in other counties' legislations; from a legal point of view, two main recal//withdrawal systems could be identified: a mandatory one (EU and China) and a voluntary one (USA, Canada and Australia); all the investigated systems have a co-operative approach between authority and food business operator, but different functions on their respective roles could be identified.

\section{Introduzione}

Il richiamo/ritiro degli alimenti è uno strumento fondamentale per la gestione dei rischi nel quadro di una risposta a situazioni di urgenza in materia di sicurezza degli alimenti (FAO/OMS, 2012). Negli Stati Uniti il numero di richiami alimentari ha avuto un incremento dovuto ad un interesse sulla sicurezza degli alimenti e alla sicurezza in generale da parte delle autorità governative. Inoltre l'attuazione di misure preventive nei confronti di atti di terrorismo, nuove e più sensibili tecniche di rilevamento di agenti patogeni nonché la fiducia nelle indagini epidemiologiche hanno contribuito a confermare questo trend (Kramer et al., 2005). Alcuni paesi si sono dotati solo recentemente di un sistema di richiamo degli alimenti; è questo il caso per esempio della Cina che sull'onda emotiva di alcuni incidenti alimentari ha introdotto nel proprio ordinamento la food safety law del 2009 in cui sono contenute le basi giuridiche per la costituzione di un sistema di richiamo (Zhang, 2010).

Il termine ritiro (in inglese withdrawal, in francese retrait) ed il termine richiamo (in inglese recall, in francese rappel) rappresentano i termini fondamentali del lessico che caratterizza il sistema di richiamo. L'impiego però di questi termini non è sempre univoco ed il significato che i termini assumono nei vari sistemi nazionali di richiamo possono avere valenze diverse. Con l'obiettivo di valutare le eventuali differenze fra i vari sistemi nazionali di richiamo degli alimenti sono state analizzate le caratteristiche salienti dei sistemi nazionali di Canada, Australia, Stati Uniti e Cina in confronto col sistema dell'Unione Europea. L'analisi era volta allindividuazione delle basi giuridiche, degli stakeholders coinvolti, delle definizioni utilizzate ai fini del ritiro e del richiamo, e dello stato giuridico di obbligatorietà 0 volontarietà dell'istituto giuridico del richiamo nel settore della sicurezza degli alimenti. Nel presente studio, sono state analizzate le normative di riferimento dell'Unione Europea e delle singole realtà nazionali prese in considerazione. Per quanto riguarda l'organizzazione sul piano amministrativo, si sono consultati i siti web ufficiali delle agenzie governative coinvolte nella gestione dei sistemi di richiamo nazionali e i siti istituzionali dei vari paesi oggetto dello studio: per il Canada il sito della Canadian Food Inspection Agency, per gli Stati Uniti i siti della Food and Drug Administration e del Food Safety and Inspection Service, per la Cina il sito del Ministero della salute, per l'Australia il sito della Food Standards Australia New Zealand Agency. È stata infine condotta una revisione perlustrativa della letteratura scientifica utilizzando la banca dati ScienceDirect per identificare gli articoli accademici che avessero come soggetto il richiamo degli alimenti: tale ricerca è stata effettuata utilizzando le seguenti parole chiave: recall, withdrawal, food, recall system, i termini sono stati utilizzati singolarmente e in combinazione fra di loro aggiungendo anche le parole Canada, China, USA, Australia, UE.

\section{Definizione di ritiro e richiamo}

Il Reg. (CE) 178/02 (Parlamento Europeo, 2002b) nonostante definisca principi, responsabilità degli operatori del settore alimentare e funzioni e compiti dell'autorità competente in caso di azioni di ritiro e richiamo di un prodotto alimentare, non definisce giuridicamente né il ritiro né il richiamo. Una definizione legale di ritiro e richiamo è rinvenibile però nella Dir. 2001/95/CE del Parlamento e del Consiglio del 3 dicembre 2001 (Parlamento Europeo, 2002a) relativa alla sicurezza generale dei prodotti (non
Correspondence: Gaetano Liuzzo, Azienda Unità Sanitaria Locale di Modena - Distretto di Carpi, Piazzale Donatori di Sangue 3, 41012 Carpi (M0), Italy.

Tel. +39.059.659949 - Fax: +39.059.659994

E-mail: g.liuzzo@ausl.mo.it

Key words: Food recall, Food withdrawal, National food recall system.

Conflict of interests: the authors declare no potential conflict of interests.

Received for publication: 28 May 2013

Accepted for publication: 22 July 2013.

This work is licensed under a Creative Commons Attribution 3.0 License (by-nc 3.0).

(C) Copyright G. Liuzzo et al., 2013

Licensee PAGEPress, Italy

Italian Journal of Food Safety 2013; 2:e47

doi:10.4081/ijfs.2013.e47

alimentari). All'art. $2 \mathrm{l}$ g) si definisce richiamo: le misure volte ad ottenere la restituzione di un prodotto pericoloso che il fabbricante o il distributore ha già fornito o reso disponibile ai consumatori, mentre alla l h) si definisce ritiro: qualsiasi misura volta a impedire la distribuzione e l'esposizione di un prodotto pericoloso, nonché la sua offerta al consumatore. A queste definizioni si è chiaramente ispirato il legislatore nazionale quando ha riportato i suddetti termini nelle linee guida ai fini della rintracciabilità degli alimenti e dei mangimi per fini di sanità pubblica (art. 3, Accordo n 2334 del 28/07/2005; Ministero Italiano della Salute, 2005). Altri paesi della Comunità Europea, attraverso documenti delle proprie agenzie nazionali per la sicurezza degli alimenti, hanno proposto definizioni diverse. La Food Safety Authority of Ireland (FSAI) definisce il withdrawal cioè il ritiro, come la rimozione di un alimento unsafe a rischio dal mercato prima che abbia raggiunto il consumatore e come richiamo - recall - la rimozione dal mercato di un alimento a rischio quando ha raggiunto il consumatore e la notificazione di questo al consumatore (FSAI, 2010). La forma può essere diversa ma di fatto nella sostanza i contenuti sono quelli originariamente stabiliti nella definizione della Direttiva comunitaria sopracitata. La stessa cosa non si può dire invece a livello internazionale dove vi sono differenze terminologiche che riflettono invece concetti differenti (Tabella 1).

\section{Il sistema di ritiro/richiamo nell'Unione Europea}

La base normativa dell'istituto giuridico del ritiro/richiamo degli alimenti è rappresentata 
dall'art. 19 del Reg. (CE) 178/2002 (Parlamento Europeo, 2002b).

Al paragrafo 1 dell'art. 19 si stabilisce che, nel caso in cui l'operatore ritenga, 0 abbia motivo di ritenere, che un alimento in qualche modo passato nella sua disponibilità perché $d a$ lui importato, prodotto, trasformato, lavorato o distribuito ma che non sia più sotto il controllo immediato di tale operatore, non sia conforme ai requisiti di sicurezza degli alimenti, tale operatore deve immediatamente avviare le procedure per ritirarlo e informare le autorità competenti. L'obbligo di ritiro dell'alimento è quindi subordinato alla non conformità del prodotto ai requisiti di sicurezza ed al fatto che lo stesso prodotto non sia più sotto il controllo immediato dell'operatore che prende coscienza e conoscenza della non conformità.

Nella seconda parte del primo paragrafo si dispone poi che se il prodotto può essere arrivato al consumatore, lo stesso operatore che ha avviato le procedure per ritirarlo ha l'ulteriore obbligo di informare i consumatori in maniera efficace e accurata, del motivo del ritiro e richiamare i prodotti già forniti ai consumatori quando altre misure siano insufficienti a conseguire un livello elevato di tutela della salute. Ciò a dire che, qualora la semplice informazione al consumatore circa il motivo del ritiro e l'eventuale misura di gestione del rischio, per esempio la cottura dell'alimento 0 la sua distruzione, fossero considerate insufficienti a garantire la tutela della sua salute, allora l'operatore ha l'obbligo di richiamare quell'alimento, cioè approfondire la sua azione di ritiro sino al consumatore.

Sono individuabili due livelli di comunicazione sul rischio a cui l'operatore sottende. Il primo è una informazione obbligatoria rivolta all'autorità competente che si concretizza nel momento della consapevolezza della non conformità dell'alimento ai requisiti di sicurezza, e la seconda altrettanto obbligatoria che si compie qualora il prodotto non conforme fosse arrivato al consumatore finale ed è a quest'ultimo rivolta.

Il dettato del secondo paragrafo dell'art. $19 \mathrm{si}$ impegna nel delineare, ritagliandoli dal più ampio contesto generale, gli specifici compiti di quell'operatore responsabile dell'attività di vendita al dettaglio o distribuzione, cioè di quei soggetti che hanno soltanto indiretta responsabilità nella circolazione del prodotto insicuro (Aversano e Pacileo, 2006), con la caratteristica di non incidere sul confezionamento, sull'etichettatura, sulla sicurezza o sull'integrità dell'alimento. Questo operatore ha l'obbligo, come già definito in modo generale al paragrafo precedente, di avviare procedure di ritiro dal mercato di quei prodotti non conformi, entro i limiti della propria attività, e di contribuire a garantire la sicurezza degli alimenti fornendo al riguardo le informazioni necessarie ai fini della loro rintracciabilità, collaborando agli interventi di responsabili della produzione, della trasformazione e della lavorazione e/o delle autorità competenti. Le predette disposizioni stabiliscono quindi le regole generali che devono rendere possibile una efficace collaborazione tra i diversi soggetti della catena alimentare (Capelli et al., 2006).

Il terzo paragrafo dell'art. 19 individua una specifica situazione in cui l'operatore del settore alimentare può trovarsi qualora ritenga 0 abbia motivo di ritenere di aver immesso sul mercato un alimento che possa essere dannoso per la salute umana. In questo caso l'operatore deve informare l'autorità competente circa gli interventi adottati per evitare i rischi al consumatore finale. Tale disposizione deve essere collegata con il disposto dell'art. 10 del Reg. (CE) 178/02 (Parlamento Europeo, 2002b), il quale prevede che l'autorità pubblica adotti a sua volta provvedimenti opportuni per informare i cittadini nel caso in cui vi siano ragionevoli motivi per sospettare che un alimento o mangime possa comportare un rischio per la salute umana o animale (Bruno, 2003). Il dovere di informazione affermato dall'art. 10, deve contemperare l'esigenza di non creare inutili situazioni di panico, che tra l'altro potrebbero comportare comportamenti non corretti da parte del consumatore (Viti, 2003). Inoltre, ai sensi dell'art. 50.2 del Reg. (CE) 178/02 (Parlamento Europeo, 2002b), è fatto obbligo all'autorità competente, qualora ... disponga di informazioni relative all'esistenza di un grave rischio, diretto o indiretto, per la salute umana dovuto ad alimenti o mangimi, trasmettere tale informazione alla Commissione nell'ambito del sistema di allarme rapido.

Gli operatori, nel caso di immissione sul mercato di un alimento dannoso per la salute, non impediscono né scoraggiano la cooperazione di chiunque con le autorità competenti.

È con clausola di chiusura che si dispone, al paragrafo quarto dell'art. 19, che gli operatori del settore alimentare collaborino ex post, ossia a rischio ormai realizzato (Bruno, 2003), con le autorità riguardo ai provvedimenti volti ad evitare o ridurre i rischi provocati da un alimento che forniscono o hanno fornito al consumo. Questa cooperazione collaborativa tra operatori ed autorità competente può riguarda$r e$ anche la consulenza da quest'ultima fornita agli operatori che necessitano di conoscere in che modo gli obblighi debbono essere ottemperati ed a loro volta le autorità competenti devono prestare assistenza agli operatori qualora questi lo richiedano (Capelli et al., 2006), sono disposizioni queste ispirate al principio di lealtà ed effettiva collaborazione che pervadono tutto l'articolo 19 (Aversano e Pacileo, 2006).

Quanto disposto dall'art. 19 che in rubrica indica obblighi relativi agli alimenti: operatori

Tabella 1. Confronto dei termini recall e withdrawal.

UE Le misure volte ad ottenere la restituzione di un prodotto pericoloso che il fabbricante 0 il distributore ha già fornito 0 reso

Qualsiasi misura volta a impedire la distribuzione e l'esposizione di un prodotto pericoloso, nonché

disponibile ai consumatori

Australia A recall is an action taken to remove from distribution, sale and consumption, food which may pose a health and safety risk to consumers

Canada (verb tense) Means for a firm to remove from further sale or use, or to correct, a marketed product that contravenes legislation administered andor enforced by CFIA. (noun tense) Denotes the process of recalling the affected product and encompasses all tiers of the affected product distribution system.

USA A firm's removal of distributed (i.e. the product has left the firm's direct control) meat or poultry products from commerce when there is reason to believe that such products are adulterated or misbranded under the provisions of the Federal Meat Inspection Act (FMIA) or the Poultry Products Inspection Act (PPIA)

la sua offerta al consumatore

is action taken to remove food from the

supply chain where there

is no public health and safety issue.

Means a firm's removal from further sale or use of a marketed product that does not violate legislation administered ACIA (2013)

A firm's removal or correction, on its own initiative, of a distributed product that involves a minor company quality program or regulatory program infract
not cause the product to be adulterated or misbranded
USDA-FSIS (www.fsis. usda.gov)

Dir. 2001/95/CE (Parlamento

Europeo, 2002a)

FSANZ (2008)

ACIA (2013)

s. 
del settore alimentare farebbe pensare che il driver di un ritiro e/o richiamo di un alimento non conforme ai requisiti di sicurezza sia solo ad esclusivo appannaggio dell'operatore del settore alimentare, in realtà l'autorità competente può imporre restrizioni all'immissione sul mercato di alimenti disponendone il ritiro sia nel caso di alimenti conformi, ex art. 14.8 del Reg. (CE) 178/02 (Parlamento Europeo, 2002b), che nel caso in cui individui una non conformità ed intervenga per assicurare che l'operatore ponga rimedio alla situazione creatasi. L'art. 54 del Reg. (CE) 882/04 (Parlamento Europeo, 2004) stabilisce infatti che Nel decidere l'azione da intraprendere, a seguito dell'individuazione di una non conformità l'autorità competente tiene conto della natura della non conformità e dei dati precedenti relativi a detto operatore per quanto riguarda la non conformità. L'azione che intraprende può essere, fra le altre, il monitoraggio e, se necessario, la decisione del richiamo, del ritiro elo della distruzione di mangimi o alimenti (art. $54 \mathrm{p} 2 \mathrm{l}$ c).

\section{Caratteristiche del sistema di ritiro/richiamo in Australia}

In Australia la Clause 12 of Standard 3.2.2 Food Safety Practices and General Requirements dispone in modo del tutto originale l'obbligo non tanto di ritiro o richiamo di un alimento pericoloso quanto l'obbligo, da parte solo di alcuni operatori del settore alimentare, di disporre di un sistema di richiamo scritto e documentato. Tale obbligo vale per commercianti all'ingrosso, produttori, e importatori di prodotti alimentari. Sono invece esen- tati dall'avere un piano di recall i rivenditori o gestori di un'attività di generi alimentari come per esempio un supermercato, un ristorante 0 un negozio take away, a meno che non siano parte integrante di una catena di commercianti all'ingrosso, produttori 0 importatori. Tale esclusione si giustifica per il fatto che gli alimenti in vendita nei ristoranti e nei negozi take away generalmente vengono consumati immediatamente, per cui in questi casi il sistema di ritiro non sarebbe pratico (ANZFA, 2001). Nel sistema australiano il richiamo è l'azione intrapresa per rimuovere dalla distribuzione, vendita e consumo gli alimenti che possono rappresentare un rischio per la salute e sicurezza dei consumatori. Il presupposto richiesto per l'attivazione di un richiamo è la ragionevole possibilità che l'utilizzo 0 il consumo dell'alimento possa causare conseguenze nocive sulla salute 0 addirittura la morte. Costituiscono motivi per attivare un recall: la contaminazione di batteri patogeni, la presenza di agenti chimici e fisici come anche l'etichettatura non conforme di prodotti che per esempio possono contenere allergeni. Quando l'operatore decide volontariamente l'attivazione di un richiamo, notifica tale azione alle istituzioni governative, incluso The Commonwealth Minister responsabile dei consumer affairs. La notifica deve avvenire secondo quanto stabilito dal Australian Trade Practices Act 1974 entro due giorni dall'inizio del richiamo. Esistono due livelli di recall: il trade recall ed il consumer recall. Il primo comporta il recupero di un prodotto alimentare che non è nella disponibilità del consumatore, dalle sedi o luoghi che non sono controllate dall'operatore che ha la responsabilità del richiamo. Di fatto questo richiamo si realizza a livello di centri di distribuzione o depositi. Il secondo tipo di recall è più estensivo nella sua azione e comporta il recupero del prodotto alimentare da tutti i punti della filiera fino al prodotto già in possesso del consumatore. In questo tipo di richiamo oltre all'informazione delle istituzioni governative è obbligo informare anche $i$ consumatori. I richiami sono volontari e si realizzano quando il cosiddetto sponsor, cioè l'operatore che ha la primaria responsabilità della catena alimentare (produttore, commercianti all'ingrosso e importatori), inizia l'azione di rimozione dalla distribuzione, vendita e/o consumo del prodotto alimentare. L'uso del termine voluntary significa che la decisione di attivare un richiamo è a discrezione dello sponsor, mentre i soggetti della filiera a valle dello sponsor sono tenuti obbligatoriamente a garantire l'azione dell'operatore che ha deciso di rimuovere dal mercato il prodotto considerato a rischio. Il richiamo può essere anche obbligatorio quando è il Commonwealth o lo Stato o il Territory Government che ordinano il richiamo di un prodotto alimentare dalla distribuzione, vendita e/o consumo. Tale eventualità ricorre nel caso in cui il prodotto richiamato rappresenti un rischio per la salute e sicurezza pubblica.

Diverso invece è il caso del ritiro di un alimento dal mercato. Questi si realizza infatti con la rimozione dalla supply chain di un alimento che non costituisce un rischio per la salute. Sono due le ragioni che giustificano un ritiro: un difetto qualitativo (che può riguardare il colore 0 il peso 0 una irregolarità dell'etichettatura) che non costituisce un rischio potenziale per la salute; e una precauzione nell'attesa della valutazione di situazioni di potenziale rischio per la salute del consumato-

Tabella 2. Paragone dei sistemi nazionali di richiamo degli alimenti.

\begin{tabular}{|c|c|c|c|c|}
\hline Paese & Tipo di richiamo & $\begin{array}{l}\text { Profondità } \\
\text { del richiamo }\end{array}$ & $\begin{array}{l}\text { Classificazione } \\
\text { del richiamo }\end{array}$ & Base legale \\
\hline UE & Obbligatorio & $\begin{array}{l}\text { Ritiro } \\
\text { Richiamo }\end{array}$ & & Art. 19 Reg.(CE) 178/2002 (Parlamento Europeo, 2002b) \\
\hline Australia & Volontario* & $\begin{array}{l}\text { Trade } \\
\text { Consumer }\end{array}$ & & $\begin{array}{l}\text { The Australia New Zealand Food Standards } \\
\text { Code clause } 12 \text { of Standard } 3.2 .2 \\
\text { Food Safety } \\
\text { Practices and General Requirements } \\
\text { (http://www.foodstandards.gov.au/Pages/default.aspx) }\end{array}$ \\
\hline Canada & Volontario* & $\begin{array}{l}\text { Distributor } \\
\text { Importer } \\
\text { Manaufacturer } \\
\text { Retailer }\end{array}$ & Classe I-II-III & $\begin{array}{l}\text { Art. } 19 \\
\text { Canadian Food Inspection Agency Act (http://www.inspection.gc.ca) }\end{array}$ \\
\hline USA & Volontario* & $\begin{array}{l}\text { Wholesale level } \\
\text { Retail level } \\
\text { HRI level } \\
\text { Consumer level }\end{array}$ & Classe I-II-III & $\begin{array}{l}\text { CFR Title } 21 \text { Volume } 1 \\
\text { FSIS DIRECTIVE } 8080.1 \text { (http:/www.fsis.usda.gov/wps/portal/fsis/home) }\end{array}$ \\
\hline Cina & Obbligatorio & & & $\begin{array}{l}\text { The Food Safety Law of the People's Republic of China } \\
\text { cap. IV art. } 53 \\
\text { cap. VII art. } 72 \\
\text { cap. IX art. } 85 \text { (Repubblica Popolare Cinese, 2009) }\end{array}$ \\
\hline
\end{tabular}

USA, United States of America; UE, Unione Europea. *Prevalentemente volontario, l'autorità può comandare un richiamo. 
re. A differenza del richiamo, il ritiro non necessita di alcuna notifica né alle autorità né al consumatore (FSANZ, 2008).

\section{Caratteristiche del sistema di ritiro/richiamo in Canada}

In Canada il regime di salubrità degli alimenti riposa sulla Loi sur les aliments et drogues che viene applicata congiuntamente da Santé Canada cioè il Ministero federale della salute e dall'Agence Canadienne d'Inspection des Aliments (ACIA). Il Ministero è incaricato di stabilire i regolamenti e le norme che riguardano la sicurezza degli alimenti, mentre l'ACIA, che dipende dal Ministero dell'Agricoltura e dell'Agroalimentare, è incaricata dell'applicazione delle norme fissate dal Santé Canada così come di vigilare sul rispetto delle leggi commerciali di alcuni prodotti alimentari. L'ACIA esercita quindi tutte le attività federali in materia di ispezione degli alimenti rappresentando un ... des rares agences au monde dont les responsabilités s'étendent sur toute la gamme du cycle alimentaire (Butler et al., 2012). Nel quadro di un processo di richiamo degli alimenti, l'ACIA collabora con il Santé Canada e con l'Agence de la Santé Publique du Canada (ASPC). L'industria alimentare canadese effettua la maggior parte dei richiami su base volontaria e si occupa altresì di verificarne l'efficacia. Tuttavia, se un impresa non ha la capacità 0 la volontà di eseguire un richiamo, il Ministero dell'Agricoltura e dell'Agroalimentare può, ai sensi del Loi sur l'Agence canadienne d'inspection des aliments, ordinare allimpresa di procedere al richiamo qualora giudicasse che il prodotto presenti un rischio per la salute pubblica, la sanità animale o la protezione dei vegetali (art. 19, Loi sur l'Agence canadienne d'inspection des aliments). Recita infatti l'art. 19 della Loi sur l'Agence canadienne d'inspection des aliments: S'il a des motifs raisonnables de croire qu'un produit régi par une loi ou disposition dont l'Agence est chargée d'assurer ou de contrôler l'application aux termes de l'article 11 présente un risque pour la santé publique ou celle des animaux ou des végétaux, le ministre peut, par avis signifié à la personne qui vend, met en marché ou distribue ce produit, en ordonner le rappel ou son envoi à l'endroit qu'il désigne (ACIA, 2013). Anche in questo sistema si distinguono due tipi di richiamo (recall in inglese, rappel in francese): quello volontario avviato ed effettuato da una impresa, e quello obbligatorio che viene attivato in virtù dell'art. 19. Con il termine di ritiro (withdrawal in inglese, retrait in francese) invece si intende, il rilevamento da parte dell'impresa dalla vendita 0 dall'utilizzazione di un prodotto commercializzato che non contravviene la legge amministrata o applicata dall'ACIA, nella sostanza la rimozione dal commercio per motivi che non sono sanitari di alimenti. Nel sistema canadese gli operatori della filiera (dettaglianti, produttori, distributori, importatori) sono parimenti coinvolti nel sistema di recall/rappel. L'ACIA svolge una funzione collaborativa con l'impresa alimentare che attiva il richiamo, verificandone l'efficacia e fornendo consulenza sulla valutazione dei rischi, a quest'ultimo proposito determinando il tipo/classificazione di richiamo. I richiami entrano in una delle seguenti classi: classe I) corrisponde ad una situazione in cui esiste una probabilità ragionevole che l'utilizzazione del prodotto alimentare non conforme 0 l'esposizione allo stesso comporta da effetti nocivi gravi sulla salute fino alla morte; classe II) è la situazione nella quale l'utilizzazione di un prodotto non conforme o l'esposizione allo stesso può comportare effetti nocivi temporanei sulla salute 0 nella quale la probabilità di effetti nocivi gravi e debole; classe III) corrisponde alle situazione dove l'utilizzazione di un prodotto non conforme o l'esposizione allo stesso non dovrebbe comportare effetti nocivi sulla salute (ACIA, 2013). Sono classificati come alerts i richiami di prodotti alimentari che possono provocare allergie e/o fenomeni allergici (ACIA, 2013).

Il produttore, importatore, dettagliante, distributore che immette sul mercato un prodotto insalubre o non conforme alle leggi e regolamenti, deve rilevarlo dal mercato cioè richiamarlo. Contestualmente deve comunicare immediatamente all'ACIA dell'azione intrapresa. L'agenzia può collaborare con l'impresa che deve informare l'ACIA di qualunque decisione che costituisce un'azione correttiva intrapresa a salvaguardia della salute e sicurezza del pubblico. A questo proposito, i coordinatori che operano nei centri operativi periferici dell'agenzia, hanno il compito di raccordare l'operato dell'azienda alimentare con le disposizioni dell'ACIA che coordina i richiami a livello centrale. L'impresa ha quindi l'obbligo di informare l'agenzia immediatamente dell'azione di richiamo ed entro due ore da tale disposizione, deve preparare un messaggio da comunicare al pubblico. Il messaggio deve però essere presentato preventivamente all'agenzia che ne valuta la forma ed i contenuti. L'ACIA ha la possibilità, qualora non ne condivida il contenuto di comunicare indipendentemente dall'impresa un messaggio rivolto al pubblico.

\section{Caratteristiche del sistema di ritiro/richiamo negli Stati Uniti d'America}

Il sistema statunitense sulla sicurezza alimentare si presenta fortemente frammentario nelle strutture. Tutte le decisioni relative alla sicurezza degli alimenti passano attraverso lo sharing delle funzioni tra ben dodici agenzie, ciascuna delle quali è affidataria di funzioni diverse, seppure ovviamente tutte riconducibili alla tutela della sicurezza degli alimenti e della salute dei cittadini (Sgueo, 2008). Tra i dodici petali della rosa, sei sono le strutture che ricoprono un ruolo principale nella realizzazione delle attività correlate alla sicurezza degli alimenti. Sul piano funzionale le strutture si differenziano per l'oggetto del provvedimento come descritto qui di seguito.

La Food and Drug Administration (FDA) ha il compito di assicurare che i prodotti alimentari, ad esclusione delle carni e del pollame, siano salubri, sani ed etichettati in modo onesto e corretto. La FDA ha però giurisdizione su alimenti che contengono meno del $3 \%$ di carni fresche 0 pollame, così come su alimenti che contengono meno del $2 \%$ di carni cotte o pollame. In riferimento alle uova è corresponsabile insieme con l'Agricultural Marketing Service (AMS) dell'ispezione nei ristoranti, negli stabilimenti di confezionamento ed in strutture simili che servono o l'alimento o utilizzano l'ingrediente nei prodotti.

Lo United States Departement of Agruculture (USDA) si occupa di regolamentazione delle carni e del pollame e, tramite il suo Food Safety Inspection Service (FSIS), sorveglia le stesse tipologie di prodotti affinchè, sia nel commercio interstatale che in quello d'esportazione, siano sicuri per il consumo umano e correttamente confezionati ed etichettati.

L'Agricultural Marketing Service (AMS) assicura le uova fresche e gli stabilimenti di prodotti derivati coinvolti nel commercio inter ed intrastatale, nonché nel flusso delle esportazioni.

Il Federal Grain Inspection Service (FGIS) ispeziona il grano, il sorgo ed il riso per le aflatossine.

L'Environmental Protection Agency (EPA) regolamenta, invece, tutti gli erbicidi in relazione ai residui nei prodotti alimentari e nei mangimi per animali.

Il National Marine Fisheries Service (NMFS) conduce ispezioni volontarie sui frutti di mare (Babuscio, 2005) Sebbene il sistema si presenti con contorni non facilmente delineabili si può in sintesi affermare che allo USDA spetta la regolamentazione delle carni e del pollame mentra alla FDA all other food product. Ed in questi ambiti sono le agenzie coinvolte nel sistema di recall degli alimenti. Con la redazione della FSIS directive 8080.1 revision 6 del 26/10/2010, Recall of Meat and Poultry products, la FSIS - ramo del United States Department of Agriculture (USDA) - è responsabile dei richiami che implicano le carni rosse e avicole, mentre la FDA degli altri alimenti, inclusi i prodotti della pesca e i prodotti in genere sulla base legale del Code of Federal Regulations (CFR Title 21, volume 1, chapter I, subchapter A, part 7, sub part C) (Kramer et al., 2005). Il richiamo di alimenti costituisce un buon esempio di strumento di 
congiunzione fra la gestione del rischio e la sua comunicazione; risulta infatti che il richiamo sia l'azione intrapresa volontariamente dall'impresa che è stata informata da una agenzia governativa 0 ha preso coscienza direttamente ed indipendentemente della possibilità di un effetto negativo sulla salute del consumatore che consuma un suo prodotto. Lo scopo di un richiamo è quello di rimuovere dal commercio un prodotto alimentare che si crede essere adulterated o misbranded. Il food safety system nord-americano ruota tutt'intorno al concetto giuridico di adulterazione: il termine, cioè, non è soltanto considerato nel suo significato come sofisticazione o falsificazione qualitativa, ma è circoscritto a ciò che la legge statunitense prevede a riguardo (Babuscio, 2005). Ciò che rende un alimento adulterated è definito in diverse norme statunitensi: Food, Drug and Cosmetic Act (21 U.S.C. §331; http://www.fda.gov), Meat Inspection Act (21 U.S.C. §610, 9 CFR 301.2), Poultry Products Inspection Act (21 U.S.C. \$458, 9 CFR 381.1) e Egg Products Inspection Act (21 U.S.C. §1037, 9 CFR 590.5) (http://www.fsis.usda.gov/wps/portal/fsis/home).

Sono considerati come aduleterated tutti gli alimenti che contengono sostanze tossiche 0 nocive per la salute 0 che contengono residui chimici di pesticidi, farmaci veterinari, additivi che li rendono a pericolosi. Sono ancora considerati adulterated gli alimenti che in tutto 0 in parte risultano insudiciati, putridi o decomposti o comunque inadatti al consumo, quelli preparati, confezionati o manipolati in condizioni non igieniche tali da poterli rendere dannosi per la salute. In sintesi, tutta una serie di situazioni in cui l'alimento può rappresentare un pericolo per la salute umana. Sono invece da considerare misbranded tutti gli alimenti la cui etichetta risulti per una serie varia di motivazioni non conforme alle norme stabilite in materia di etichettatura. Non necessariamente la non conformità rappresenta 0 può rappresentare un problema di natura sanitaria (Food, Drug and Cosmetic Act: 21 U.S.C. §343; http://www.fda.gov).

La natura volontaria del processo è la principale differenza fra il richiamo condotto dal produttore e/o detentore di alimenti rispetto al richiamo imposto dallo FSIS per esempio a seguito del sequestro di un prodotto alimentare. Il richiamo di alimenti differisce inoltre dal ritiro dal mercato e dallo stock recovery queste ultime due azioni non comprendono casi di alimenti adulterated 0 misbranded. Il ritiro dal mercato è la rimozione volontaria di prodotti alimentari che non violano alcun atto imposto dallo FSIS e/o dalla FDA, mentre lo stock recovery consiste nella rimozione o correzione da parte dell'azienda di un alimento che non è stato commercializzato 0 che si trova ancora sotto il diretto controllo della stessa.

Il governo classifica i richiami in tre gruppi generali di rischio: classe I) corrisponde ad una situazione dove esiste una probabilità ragionevole che l'utilizzazione di un prodotto non conforme 0 una esposizione a questi comporterà degli effetti nocivi gravi sulla salute fino alla morte. Diverse possono essere le cause di un richiamo di classe1, l'USDA include Listeria monocytogenes, E.coli 0157:H7, un allergene non dichiarato, Salmonella spp., materiali estranei; classe II) corrisponde ad una situazione nella quale l'utilizzazione di un prodotto non conforme o l'esposizione a questi può comportare degli effetti nocivi temporanei sulla salute 0 nella quale la probabilità di effetti nocivi gravi è scarsa. In questa classe si possono includere allergeni minori, etichettatura non corretta per quanto riguarda gli ingredienti; classe III) corrisponde ad una situazione dove l'utilizzazione di un prodotto non conforme o l'esposizione a questi non dovrebbe comportare degli effetti nocivi sulla salute.

La terminologia definita dallo FSIS (sovrapponibile a quella della FDA) è la seguente:

Recall (richiamo): la rimozione dal commercio di un prodotto (carne e prodotti avicoli) non più sotto il diretto controllo dell'azienda quando si ha ragione di credere che quel prodotto sia adulterated o misbranded sulla base del Federal Meat Inspection Act (FMIA) 0 del Poultry Products Inspection Act (PPIA). Il richiamo non include ne il ritiro dal mercato ne lo stock recovery.

Market withdrawal (ritiro dal mercato): la rimozione o correzione, di propria iniziativa, da parte dell'azienda, di un prodotto distribuito che comporta l'infrazione minore del programma qualità di una azienda 0 di un programma di regolazione che comunque non è causa di un prodotto adulterated 0 misbranded. Per esempio un prodotto che non è conforme agli standard qualitativi dell'azienda che lo riceve perché scolorito.

In base alla profondità 0 estensione del richiamo lo FSIS distingue:

Wholesale level: livello di ingrosso, il prodotto è stato distribuito a livello di Magazzino e/o deposito o di centro di distribuzione dove comunque non è sotto il controllo diretto dell'azienda che lo ha prodotto. È questo il livello di distribuzione che sta fra il produttore e il dettagliante.

Retail level: commercio al dettaglio. Il prodotto è stato ricevuto dal dettagliante per la vendita al consumatore ma non è stato ancora venduto al consumatore.

Hotel restaurant institutional (HRI) level: il prodotto è stato ricevuto dagli hotels, ristoranti ed altre clienti istituzionali.

Consumer level: il prodotto è stato venduto al consumatore domestico anche se quantità identificabili possono essere rimaste sotto il controllo del dettagliante.

Anche nel sistema americano le agenzie hanno una funzione fondamentale nella verifi- ca dell'efficacia del richiamo nonché nell'informazione al consumatore.

\section{Caratteristiche del sistema di ritiro/richiamo in Cina}

In Cina il sistema di gestione del controllo degli alimenti coinvolge a livello centrale lo State Council's Food Safety Committee, il Ministry of Health (MOH), il Ministry of Agriculture (MOA), l'Administration of Quality Supervision, Inspection and Quarantine Department (AQSIQ), l'Industry and Commerce Department (IAC) e il State Food and Drug Administration Department (SFDA). Localmente invece distinguiamo una serie di Dipartimenti provinciali, di Uffici nelle città e nelle regioni (Jia e Jukes, 2013). La normativa cinese in materia di alimenti può essere divisa in tre principali livelli. Il primo livello è rappresentato dalle basic laws: la Food Safety Law (2009), la Agri-food Quality and Safety Law (2006), il Product Quality Law (2000), la Agriculture Law (2003), la Law of Standardization (1989), la Law on the Inspection of Import and Export Commodities (2002) (www.procedurallow.cn). Queste norme sono il prodotto dell'attività del National People's Congress e sono prevalenti rispetto alle norme dei livelli successivi. Il secondo livello normativo è costituito da leggi e regolamenti come la Supervision Methods of Quality and Safety in Food Enterprises, la Regulation of Food Labels, l'Administrative Regulation of Food additives ed un gran numero di standard nazionali che stabiliscono i requisiti di sicurezza degli alimenti. Il terzo ed ultimo livello è rappresentato da vari regolamenti che sono promulgati dai governi delle provincie come per esempio l'Administrative measures of local food safety standards of Shanghai (Jia e Jukes, 2013). È con la Food Safety Law (Repubblica Popolare Cinese, 2009) che si istituisce il sistema di richiamo, all'art. 53 infatti si stabilisce che: Where a food producer finds that any food it produces does not conform to the food safety standards, it shall promptly stop the production, recall all the food already placed on market for sale, notify the related producers, business operators and consumers and record the recall and notification information. Il sistema di richiamo obbligatorio prevede che, qualora il produttore di alimenti scopra che l'alimento da lui prodotto non sia conforme agli standards di sicurezza, immediatamente ne interrompe la produzione e con la stessa immediatezza richiama tutti i prodotti già sul mercato per la vendita, informando gli operatori collegati, gli operatori commerciali e i consumatori.

Se è l'operatore commerciale ad accorgersi della non conformità, questi ha l'obbligo di 
interrompere la vendita del prodotto, informare il produttore di quell'alimento, gli operatori commerciali collegati ed ovviamente i consumatori. Ma è solo se l'operatore commerciale lo considera necessario che procede al richiamo del prodotto non conforme. Sarà infatti compito del produttore gestire il richiamo e tutte le operazioni concernenti il trattamento del prodotto. Il richiamo è quindi obbligatorio per il produttore dell'alimento il quale avrà anche l'onere di fare relazione del richiamo e delle azioni correttive al Quality Supervision Department (AQSIQ) 0 alle autorità locali.

Non appena le autorità locali ricevono la notizia di un problema di sicurezza degli alimenti congiuntamente con l'Agriculture Administrative Department, il Quality Supervision Department, l'Industry and Commerce Administrative Department e il Food and Drug Supervision and Administration Department per prendere qualsiasi iniziativa volta alla gestione del rischio. Fra le azioni che sono indicate dall'art. 72 della Food Safety Law (Repubblica Popolare Cinese, 2009) vi sono: il sequestro dell'alimento e della materie prime impiegate, l'ispezione immediata dello stabilimento di produzione, l'ordine di richiamo sia al produttore che al commerciante, l'interruzione della produzione e la distruzione del prodotto alimentare contaminato e delle materie prime impiegate.

\section{Discussione}

Sul piano terminologico è evidente che, a differenza di tutti gli altri paesi presi in considerazione nell'indagine, i termini di ritiro withdrawal - e richiamo - recall - sono utilizzati nell'UE per definire la profondità dell'azione di ritiro lungo la catena alimentare. Manca, nel corpus normativo del pacchetto igiene europeo, una definizione legale di ritiro e/o richiamo. Il termine recall indica in tutti $\mathrm{i}$ modelli extracomunitari esaminati il ritiro di prodotti che sono a rischio mentre il termine withdrawal è utilizzato per individuare l'azione di ritiro di un alimento per motivi che non sono sanitari, legati per esempio alla qualità merceologica dell'alimento stesso. Sia negli Stati Uniti che in Canada il termine alert (allerta) è utilizzato nell'ambito dei richiami di prodotti alimentari che possono provocare allergie e/o fenomeni allergici.

Sul piano giuridico, se per gli operatori del settore alimentare della Cina e dell'UE le azioni di ritiro/richiamo sono comunque obbligatorie, negli altri paesi il regime è prevalentemente volontario. In questi paesi sono le imprese stesse che decidono se procedere 0 meno ad una azione di richiamo, fermo restando la possibilità da parte delle autorità di definirne, qualora ve ne fossero i presupposti, l'obbligato- rietà. Vale per tutti i paesi analizzati la tendenza al coinvolgimento, sia esso obbligatorio 0 volontario, di tutti gli operatori del settore alimentare. Una particolarità è rappresentata dall'Australia dove si impone l'obbligo di disporre di un piano di recall da parte di alcuni operatori e cioè grossisti, produttori e importatori, mentre l'azione di richiamo rimane su base volontaria.

La volontarietà del sistema non esclude l'obbligo di informazione immediata dell'azione di richiamo all'autorità competente, il principio è condiviso e più 0 meno regolato - entro due giorni nel sistema australiano - da tutti i paesi oggetto dello studio.

Per quanto riguarda invece la comunicazione sul rischio rivolta al consumatore, nel modello europeo l'operatore del settore alimentare ne è responsabile in modo del tutto autonomo, a differenza del modello canadese e americano dove l'autorità competente coadiuva l'operatore nella fase di comunicazione. In Canada il messaggio oggetto della comunicazione sul rischio deve essere preventivamente valutato dall'Agenzia Canadese per la Sicurezza degli Alimenti nella forma e nei contenuti.

Singolare nel modello cinese la previsione normativa che impone all'operatore l'interruzione immediata della produzione dell'alimento oggetto di richiamo.

Diverse sono le sfumature che caratterizzano invece il ruolo e le funzioni delle autorità competenti nei diversi modelli, tutti ispirati al principio della collaborazione. Le autorità competenti canadesi ed americane inoltre si fanno carico dell'informazione rivolta al consumatore, mentre nel modello europeo tale onere rimane a carico dell'impresa che ha attivato l'azione di ritiro e/o richiamo. Nei sistemi del Nord America emerge inoltre il compito esperito dalle autorità, della valutazione qualitativa del tipo di richiamo in classi I-II-III a seconda della gravità dei probabili effetti nocivi sulla popolazione per il consumo degli alimenti soggetti al ritiro.

\section{Conclusioni}

Nel contesto europeo, occorre distinguere chiaramente tra il Sistema di Allarme Rapido (SAR) per gli alimenti ed i mangimi e l'obbligo di ritiro e/o richiamo degli operatori di cui agli artt. 19 e 20 del Reg. (CE) 178/02 (Parlamento Europeo, 2002b; Capelli et al., 2006). Il SAR riguarda soltanto le autorità competenti. Ciò non toglie che da una iniziativa di ritiro e/o richiamo dell'operatore del settore alimentare, possa scaturire, a seguito della opportuna valutazione dell'autorità competente, l'attivazione della cosiddetta allerta così come disposto dall'art. 50.2 del Reg. (CE) 178/02 (Parlamento
Europeo, 2002b).

Nel settore alimentare, alle tradizionali regole di prodotto e di produzione che si traducono in requisiti fisico-chimici dei prodotti, si accompagnano un insieme di regole che attiene al modo stesso di essere e fare impresa. $\mathrm{Si}$ possono distinguere: regole di organizzazione, di relazione e di responsabilità. Il legislatore europeo ha preso atto che la sicurezza alimentare non si esaurisce nella produzione e distribuzione. L'atto negoziale della vendita non esaurisce l'area delle attività dell'impresa alimentare; le regole necessariamente investono il prima, la comunicazione rivolta al consumatore, e il dopo, l'attenzione al prodotto anche dopo la sua immissione nel mercato. Sono regole di relazione anche quelle di cui agli artt. 19 e 20 quanto agli obblighi di monitoraggio e di ritiro dei prodotti già immessi sul mercato. L'impresa alimentare non può disinteressarsi del prodotto dopo l'alienazione, ma è tenuta ad esercitare una vigilanza sull'intero mercato su cui opera, e nello stesso tempo deve esercitare la sua relazione con il mercato, attraverso una comunicazione rispettosa di regole, che sono anzitutto regole di verità e non decettività (Albisinni, 2009).

Il richiamo di alimenti costituisce un buon esempio di strumento di congiunzione fra la gestione del rischio e la sua comunicazione, risulta infatti essere l'azione intrapresa volontariamente od obbligatoriamente dall'impresa che è stata informata da una agenzia governativa 0 ha preso coscienza direttamente ed indipendentemente della possibilità di un effetto negativo sulla salute del consumatore che consuma un suo prodotto. L'indagine svolta ha messo in evidenza come il sistema sia stato codificato nei vari paesi con diverse valenze. La principale differenza fra i sistemi analizzati risiede nella terminologia utilizzata e soprattutto nella obbligatorietà 0 volontarietà dell'azione da parte dell'operatore del settore alimentare (Tabella 2).

\section{Bibliografia}

ACIA, 2013. [Canadian Food Inspection Agency/Agence Canadienne d'Inspection des Aliments]. [Sito in inglese/francese]. Disponibile al sito: http://www.inspection.gc.ca

Albisinni F, 2009. Strumentario di diritto alimentare europeo. UTET giuridica, Torino.

ANZFA, 2001. [Australia New Zealand Food Autority]. [Sito in inglese]. Disponibile al sito: http://www.foodstandards.gov.au/ Pages/default.aspx

Aversano F, Pacileo V, 2006. Prodotti alimentari e legislazione. Nuovi obblighi per le imprese. Ruolo del controllo ufficiale. Edagricole-Edizioni Agricole de Il Sole 24 
ORE Editoria Specializzata s.r.l, Bologna.

Babuscio T, 2005. Alimenti sicuri e diritto. Analisi di problemi giuridici nei sistemi amministrativi delle autorità per la sicurezza alimentare europee e statunitense. Giuffrè Editore, Milano.

Bruno F, 2003. Art. 19. In: La sicurezza alimentare nell'Unione Europea. Commentario, estratto da Le Nuove Leggi Civili commentate, anno XXVI, 1-2 gennaio-Aprile 2003. Cedam, Padova, pp 278-9.

Butler M, Norris S, Coulibaly A, Forge F, 2012. [Project de Loi S-11: loi sur la salubrité des aliments au Canada]. [Volume in francese]. Bibliothèque du Parlement ed., Ottawa, Canada.

Capelli F, Klaus B, Silano V, 2006. Nuova disciplina del settore alimentare e autorità europea per la sicurezza alimentare. Giuffrè Editore, Milano.

FA0/OMS, 2012. [Guide FA0/OMS pour l'élaboration et l'amélioration des systèmes de rappel et de suivi des aliments au niveau national]. [Volume in francese]. Organisation des Nations Unies pour l'Alimentation et l'Agriculture et Organisation mondiale de la Santé ed., Roma.

FSAI, 2010. [Guidance Note No. 10. Product recall and traceability (Revision 2)]. [Volume in inglese]. Food Safety Authority of Ireland ed., Dublino, Irlanda. Disponibile al sito: http://www.fsai.ie
FSANZ, 2008. [Industry recall protocol. A guide to conducting a food recall and writing a food recall plan]. [Volume in inglese]. Food Standards Australia New Zealand ed., Canberra, Australia.

Jia C, Jukes D, 2013. [The national food safety control system of China. A systematic review]. [Articolo in inglese]. Food Control 32:236-45.

Kramer MN, Coto D, Weindner JD, 2005. [The science of recalls]. [Articolo in inglese]. Meat Sci 71:158-63.

Ministero Italiano della Salute, 2005. Accordo, ai sensi dell'articolo 4 del decreto legislativo 28 agosto 1997, n. 281, tra il Ministro della salute e i Presidenti delle Regioni e delle Province autonome sul documento recante «Linee guida ai fini della rintracciabilità degli alimenti e dei mangimi per fini di sanità pubblica», volto a favorire l'attuazione del regolamento (CE) n. 178 del 2002 del Parlamento e del Consiglio del 28 gennaio 2002, Rep. atti n. 2334. In: Gazzetta Ufficiale n. 294, 19/12/2005.

Parlamento Europeo, 2002a. Direttiva del Parlamento Europeo e del Consiglio del 3 dicembre 2001 relativa alla sicurezza generale dei prodotti, 2001/95/CE. In: Gazzetta Ufficiale, L 11/4, 15/01/2002.

Parlamento Europeo, 2002b. Regolamento del Parlamento Europeo e del Consiglio del 28 gennaio 2002 che stabilisce i principi e i requisiti generali della legislazione ali- mentare, istituisce l'Autorita europea per la sicurezza alimentare e fissa procedure nel campo della sicurezza alimentare, 178/2002/CE. In: Gazzetta Ufficiale, L 31/1, 01/02/2002.

Parlamento Europeo, 2004. Regolamento del Parlamento Europeo e del Consiglio del 29 aprile 2004 relativo ai controlli ufficiali intesi a verificare la conformita alla normativa in materia di mangimi e di alimenti e alle norme sulla salute e sul benessere degli animali, 882/2004/CE. In: Gazzetta Ufficiale, L 191, 28/05/2004.

Repubblica Popolare Cinese, 2009. [Food Safety Law]. [Pagina in inglese]. Disponibile al sito: http://www.leggicinesi.it/view_doc.asp?docID=705

Sgueo G, 2008. Il patchwork statunitense sulla sicurezza alimentare. Disponibile al sito: http://www.diritto.it/docs/26154-il-patchwork-statunitense-sulla-sicurezza-alimentare

Viti D, 2003. Artt. 9 e 10. In: La sicurezza alimentare nell'Unione Europea. Commentario, estratto da Le Nuove Leggi Civili commentate, anno XXVI, 1-2 gennaioAprile 2003. Cedam, Padova, p 227.

Zhang Y, 2010. [Reflection and perfection of the food recall system in China]. [Articolo in inglese]. Agriculture and Agricultural Science Procedia 1:483-87. 